\title{
The Relationship Between Chronic Inflammation and Glucidic-Lipidic Profile Disorders in Kidney Transplant Recipients
}

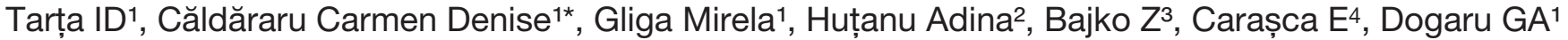 \\ 1 University of Medicine and Pharmacy Tirgu-Mures, Nephrology Department, County Clinic Hospital of Tirgu Mures \\ 2 University of Medicine and Pharmacy Tirgu-Mures, Central Laboratory, Emergency Hospital of Tirgu Mures \\ ${ }^{3}$ University of Medicine and Pharmacy Tirgu-Mures, Ist Neurology Clinic, Emergency Hospital of Tirgu Mures \\ 4 University of Medicine and Pharmacy Tirgu-Mures, IVth Internal Medicine Clinic, County Clinic Hospital of Tirgu Mures
}

\begin{abstract}
Introduction: Chronic inflammation has a proven role in atherogenesis, lipid profile parameters being related to cytokine production. In kidney transplant recipients, interleukin 6 (IL-6) is significantly associated with graft-related outcomes and also alterations of cholesterol and triglyceride metabolism. The aim of this study was to investigate the relationship between chronic inflammation and glucidic-lipidic metabolism disorders in a group of patients with kidney transplantation as renal replacement therapy. Methods: A prospective observational study which enrolled thirtysix non-diabetic kidney transplant recipients was conducted in the Nephrology and Peritoneal Dialysis Department, County Clinic Hospital of Tirgu Mures. The study group was divided as following: recipients with serum IL-6 concentration higher than 3.8 pg/ml (group A) and IL-6 within the normal range (group B). Results: Allograft recipients with higher serum IL-6 had significant higher erytrocyte sedimentation rate(ESR, $\mathrm{p}=0.0067)$. Patients with over-the-range levels of IL-6 had significant higher levels of serum cholesterol and LDL-cholesterol respectively ( $p=0.0242$ and $p=0.0081$ ). Serum Apo-B was also significant higher in Group A than Group B. Protein excretion was significant higher in patients from group A ( $\mathrm{p}=0.0013$ ). No statistical significant relationship could be proven between elevated levels of IL-6 and hbA1c, insulin and glycosuria disturbances in the two groups. Also, we found no statistical significant association between resistivity and pulsatility indices (both hilum and intragraft) or carotid intima media thickness. Conclusion: Serum interleukin 6 is related to lipid profile disorders and less to glucidic metabolism anomalies in non-diabetic kidney transplant recipients.
\end{abstract}

Keywords: inflammation, kidney transplant, interleukin 6, lipid profile disorders, proteinuria

Received: 23 August 2015 / Accepted: 21 September 2015

\section{Introduction}

Chronic inflammation and the response of immune system have a proven role in atherosclerosis development $[1,2]$. As an example, in maintaining hemodialysis patients, lipid profile parameters were related to interleukin 6 (IL-6) [3]. Allograft recipients, by receiving a non-self organ, have an aditional mechanism of activation [4-7]. Cytokines can induce production of IL-6, a key marker in the coordination of the immune response, and also increases in acute phase inflamatory markers $[8,9]$.

Increases in IL- 6 production alters triglyceride metabolism leading to hypertriglyceridemia and elevated levels of very low density lipoprotein (VLDL) cholesterol due to excessive synthesis or decrease in VLDL clearance [10]. Moreover, the effect of cytokines on triglyceride metabolism seems not to be mediated by hormones such as insulin. A study performed by Ettinger et al, has shown that IL-6 increases cholesterol synthesis and decreases cholesterol secretion [11].

In kidney transplant recipients, IL-6 is significantly associated with graft-related outcomes [12].

The aim of this study was to investigate the relationship between chronic inflammation and glucidic-lipidic

* Correspondence to: Carmen Denise Căldăraru

E-mail: caldararuc@yahoo.com metabolism disorders in a group of patients with kidney transplantation as renal replacement therapy.

\section{Methods}

A prospective observational study which enrolled thirtysix non-diabetic kidney transplant recipients was conducted in the Nephrology and Peritoneal Dialysis Department, County Clinic Hospital of Tirgu Mures, between May 1st 2014 and August 1st 2015. Demographic, transplant related data and relevant laboratory data were collected. All of these patients have performed oral glucose tolerance test (OGTT) prior to inclusion.

Glycated haemoglobin (HbA1c), blood serum high sensitive C-reactive protein (hsCRP) and Apolipoprotein B (Apo-B) were determined using the immunoturbidimetric method (Roche Romania SRL on behalf of RocheDiagnostics, Basel, Switzerland). Blood serum interleukin 6 and insulin were assesed using the chemiluminescence immunoassay method (IMMULITE 1000 Immunoas- $^{\oplus}$ say System, Siemens Healthcare GmbH, Henkestr. 127, 91052 Erlangen, Germany).

In order to compare glucidic-lipidic parameters and their relationship with chronic inflammation, the study group was divided as following: recipients with serum IL-6 concentration higher than $3.8 \mathrm{pg} / \mathrm{ml}$ (group A) and IL-6 within the normal range (group B). 
Glomerular filtration rate was estimated usind the Modified Diet in Renal Disease equation (MDRD) $[13,14]$.

Hilum resistivity (RI) and pulsatility (PI) indices were obtained by transplanted kidney Doppler ultrasound examination. Intrarenal RI and PI were considered as an average value of the measurements performed by the same physician at three different intra-graft levels (cranial, medium and caudal).Carotid intima-media thickness (CIMT) was assesed via Doppler ultrasound by the same neurologist, as a mean value for twelve measurements (three horizontallateral and three vertical-anterior assessments each side).

The study protocol and design were approved by the Ethics Committees of both County Clinic Hospital and University of Medicine and Pharmacy in Tirgu Mures. All patients have provided written informed consent prior to enrollment in this study.

Statistical analysis was performed using Graph Pad Prism 6.0 and statistical significance was defined as $\mathrm{p}<0.05$.

\section{Results}

Thirtysix adult kidney allograft recipients with no active infection or neoplastic disease and negative OGTT for diabetus mellitus were included in our study. We selected only patients with at least 6 months elapsed from surgery, the number of cases and transplantation period, is shown in Figure 1.

Main characteristics of the study group are shown in Table I. Our study population was mainly male gender $(69.44 \%)$ and renal allograft prelevation was from brain dead donors in $52.77 \%$ of the cases. The most frequent underlying cause for renal replacement therapy was represented by glomerular nephropathies $(52.77 \%)$. Average serum creatinine at inclusion was $1.653 \pm 0.8185 \mathrm{mg} / \mathrm{dl}$, and average weight $78.19 \pm 18.31 \mathrm{~kg}$.

Comparative analysis between the two groups (group A and group B) is shown as mean \pm SEM (standard error of mean) in Table II.

The analysis between the two groups shows that recipients with higher serum IL-6 had significant higher erytrocyte sedimentation rate (ESR, $\mathrm{p}=0.0067)$, with no other statistical significant relationship between elevated IL-6 and other inflammatory markers. In the same time, although patients in group A had elevated creatinine levels,

\section{Renal Allograft Survival}

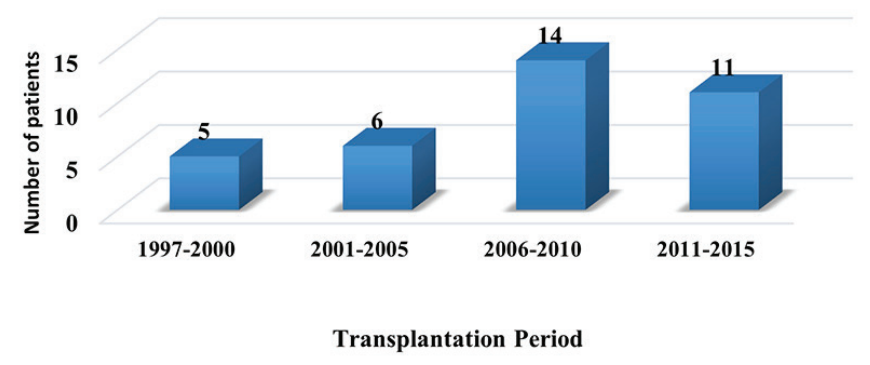

Fig. 1. Renal Allograft Survival based on transplantation period no statistical signifficant association could be established, except for estimated glomerular filtration rate using 4 variables MDRD formula, which was significant lower in recipients with higher IL-6 levels in group A.

Table I. Main characteristics of the study group

\begin{tabular}{lc}
\hline Group Characteristics at inclusion & $\begin{array}{c}\mathrm{n}=36 \\
\text { (mean } \pm \mathrm{SD}, \% \text { from total) }\end{array}$ \\
\hline Age (years) & $43.64 \pm 10.79$ \\
Weight (kg) & $78.19 \pm 18.31$ \\
Gender & \\
Male & $25(69.44 \%)$ \\
Female & $11(30.56 \%)$ \\
Donor Type & \\
Brain Dead & $19(52.77 \%)$ \\
Living related & $16(47.23 \%)$ \\
Graft positioning & \\
Left lliac Fossa & $12(33.33 \%)$ \\
Right Iliac Fossa & $24(66.67 \%)$ \\
Underlying Disease that required RRT & \\
Glomerular & $19(52.77 \%)$ \\
Tubulo-interstitial & $2(5.55 \%)$ \\
Cystic & $5(13.88 \%)$ \\
Reflux/congenital nephropathies & $4(11.15 \%)$ \\
LupusErythematosus & $1(2.77 \%)$ \\
Other & $5(13.88 \%)$ \\
Creatinine (mg/dl) & $1.653 \pm 0.8185$ \\
\hline
\end{tabular}

${ }^{*}$ RRT - renal replacement therapy

Table II. Comparative analysis between groups

\begin{tabular}{|c|c|c|c|}
\hline Studied Parameter & $\begin{array}{c}\text { Group A } \\
\text { mean } \pm \text { SEM }\end{array}$ & $\begin{array}{c}\text { Group B } \\
\text { mean } \pm \text { SEM }\end{array}$ & $\begin{array}{l}\text { Statis- } \\
\text { tical }\end{array}$ \\
\hline Age (years) & $44.86 \pm 4.753$ & $43.34 \pm 1.957$ & NS \\
\hline Hemoglobin (g/dL) & $13.11 \pm 0.6355$ & $13.25 \pm 0.4181$ & NS \\
\hline Hematocrit (\%) & $38.80 \pm 1.900$ & $38.64 \pm 1.211$ & NS \\
\hline $\operatorname{RDW}(\%)^{\star}$ & $13.52 \pm 0.4971$ & $12.82 \pm 0.2025$ & NS \\
\hline Glucose (mg/dL) & $90.43 \pm 3.689$ & $90.88 \pm 2.169$ & NS \\
\hline $\mathrm{ESR}(\mathrm{mm} / \mathrm{h})$ & $32.57 \pm 7.668$ & $14.34 \pm 2.521$ & 0.0067 \\
\hline Urea (mg/dL) & $67.43 \pm 8.552$ & $53.76 \pm 5.224$ & NS \\
\hline Creatinine (mg/dL) & $2.021 \pm 0.3164$ & $1.563 \pm 0.1491$ & NS \\
\hline $\mathrm{eGFR}^{\star \star}\left(\mathrm{ml} / \mathrm{min} / 1.73 \mathrm{~m}^{2}\right)$ & $39.20 \pm 5.771$ & $58.28 \pm 3.899$ & 0.0308 \\
\hline Uric acid (mg/dL) & $7.400 \pm 0.3066$ & $7.347 \pm 0.4037$ & NS \\
\hline Fibrinogen (mg/dL) & $317.5 \pm 13.37$ & $281.0 \pm 11.03$ & NS \\
\hline hsCRP (mg/L) & $4.701 \pm 0.9895$ & $2.518 \pm 1.069$ & NS \\
\hline Iron (mcg/dL) & $74.00 \pm 7.662$ & $88.20 \pm 10.64$ & NS \\
\hline Serum cholesterol (mg/dL) & $223.0 \pm 19.70$ & $179.5 \pm 7.766$ & 0.0242 \\
\hline Triglycerides (mg/dL) & $167.3 \pm 46.17$ & $130.7 \pm 14.53$ & NS \\
\hline HDL-cholesterol (mg/dL) & $46.69 \pm 5.013$ & $58.24 \pm 10.01$ & NS \\
\hline LDL-cholesterol (mg/dL) & $142.9 \pm 11.68$ & $104.6 \pm 6.128$ & 0.0081 \\
\hline VLDL-cholesterol (mg/dl) & $33.46 \pm 9.234$ & $26.12 \pm 2.910$ & NS \\
\hline intact-PTH* $(p g / m L)$ & $153.6 \pm 97.65$ & $75.92 \pm 12.73$ & NS \\
\hline APO-B (g/L) & $1.200 \pm 0.1234$ & $0.9107 \pm 0.05962$ & 0.0385 \\
\hline Serum proteins (g/dL) & $7.085 \pm 0.2443$ & $6.956 \pm 0.1031$ & NS \\
\hline Albumin $(g / L)$ & $41.68 \pm 1.323$ & $42.13 \pm 0.8446$ & NS \\
\hline Proteinuria (g/day) & $1.313 \pm 0.4972$ & $0.3592 \pm 0.06864$ & 0.0013 \\
\hline Glycosuria (g/day) & $0.03243 \pm 0.01610$ & $0.06490 \pm 0.03714$ & NS \\
\hline HBA1C(\%) & $5.171 \pm 0.1340$ & $5.186 \pm 0.07456$ & NS \\
\hline Insulin (uUI/mL) & $11.79 \pm 3.322$ & $10.20 \pm 1.210$ & NS \\
\hline Hilum-RI & $0.6775 \pm 0.05089$ & $0.6757 \pm 0.01574$ & NS \\
\hline Hilum-PI & $1.278 \pm 0.1926$ & $1.284 \pm 0.07827$ & NS \\
\hline Intragraft-RI & $0.6525 \pm 0.05735$ & $0.6448 \pm 0.01478$ & NS \\
\hline Intragraft-PI & $1.188 \pm 0.1993$ & $1.176 \pm 0.05430$ & NS \\
\hline CIMT (mm) & $0.5942 \pm 0.09314$ & $0.5516 \pm 0.02986$ & NS \\
\hline
\end{tabular}

* RDW - red cell distribution width, PTH - parathormone

** estimated glomerular filtration rate by 4 variables MDRD formula $\mathrm{NS}=$ not significant 
Patients with over-the-range levels of IL-6 had significant higher levels of serum cholesterol and LDL-cholesterol respectively $(\mathrm{p}=0.0242$ and $\mathrm{p}=0.0081)$. Serum Apo-B was also significant higher in Group A than Group B. No association could be made between other lipid profile parameters in the upper mentioned groups.

Protein excretion was significant higher in patients from group A ( $\mathrm{p}=0.0013)$.

In terms of glucidic metabolism, no statistical significant relationship could be proven between elevated levels of IL- 6 and hbA1c, insulin and glycosuria disturbances in the two groups.

Also, we found no statistical significant association between resistivity and pulsatility indices (both hilum and intragraft) or carotid intima media thickness.

\section{Discussions}

Renal replacement therapy by kidney transplantation is currently the best choice for the treatment of end stage kidney disease, particularly because of great improvements in patient outcome and long term graft survival. Interleukin 6 is known for it's pro-inflammatory potential in renal transplant recipients, findings in several studies have shown that there might be an association between elevated IL-6 and allograft rejection $[15,16]$ and lipid profile abnormalities, although the relationship between IL- 6 and lipid metabolism is not yet fully clarified $[9,10,17]$. The aim of our study was to investigate the relationship between IL-6 levels and glucidic-lipidic profile disorders in a group of renal transplant recipients.

In our study, IL-6 seems to be linked to increases in acute phase reactants, patients with over-the-range serum IL-6 have higher ESR, hsCRP and fibrinogen, the relationship being statistically significant only in the case of ESR.

Furthermore, patients with elevated IL-6 had significant higher levels of serum cholesterol and LDL-cholesterol, which is in agreement with other studies performed on solid organ transplantation $[17,18]$, a decrease in peripheral lipoprotein lipase being related to increasing serum lipids, as findings of other several studies have shown $[19,20]$. Serum Apo-B was also significant higher in the group of recipients with higher IL-6.

Urinary protein excretion was significant higher in patients with elevated IL-6 in our study, a statistical significant lowering in eGFR in this group being also encountered, fact that can point to a possible connection between inflammation and proteinuria, findings in a recent study showing that pro-inflammatory status can lead to higher excretion of proteinuria in hematopoietic cell transplant recipients [21].

The main limitation of our study is the number of patients, thus we consider that further studies are needed, on larger heterogenous groups of solid organ recipients, in order to clarify the relationship between pro-inflammatory cytokines and gluco-lipidic disorders.

\section{Conclusions}

Serum interleukin 6 is related to lipid profile disorders (increases in serum total cholesterol, LDL-cholesterol and apolipoprotein B) and less to glucidic metabolism anomalies in non-diabetic kidney transplant recipients. Also, elevation in serum IL-6 can lead to increasing protein excretion.

\section{Aknowledgment}

This paper was published under the frame of European Social Found, Human Resources Development Operational Programme 2007-2013, project no. POSDRU/159/1.5/S/136893.

\section{References}

1. Kaysen GA. The microinflammatory state in uremia: Causes and potential consequences. J Am Soc Nephrol 2001;12:1549-1557.

2. Willerson JT, Ridker PM. Inflammation as a cardiovascular risk factor. Circulation 2004;109: II2-II10.

3. Psychari SN, Sinos L, latrou C, Liakos G, Apostolou TS. Relations of inflammatory markers to lipid levels and autonomic tone in patients with moderate and severe chronic kidney disease and in patients under maintenance hemodialysis. Clin Nephrol. 2005;64(6):419-27.

4. Vazquez MA, Jeyarajah DR, Kielar ML, Lu CY. Long-termoutcomes of renaltransplantation: A result of the originalendowment of the donor kidney and the inflammatoryresponse to both alloantigens and injury. Curr Opin NephrolHypertens 2000;9:643-648.

5. Gotsman I, Grabie N, Gupta R, et al. Impaired regulatory T-cell response and enhanced atherosclerosis in the absence of inducible costimulatorymolecule. Circulation 2006;114:2047-055.

6. Martinez-Castelao A, Sarrias X, Bestard O,et al. Arterial elasticity measurement in renal transplant patientsunder anticalcineurin immunosuppression. TransplantProc.2005;37:3788-3790.

7. Abedini S, Holme I, Marz W et al. Inflammation in renal transplantation. Clin J Am Soc Nephrol 2009;4:1246-1254.

8. Hansson GK. Inflammation, atherosclerosis, and coronary artery disease. N Engl J Med 2005;352:1685-1695.

9. Khovidhunkit W, Kim MS, Memon RA, et al. Effects of infection and inflammation on lipid and lipoprotein metabolism: mechanisms and consequences to the host. J. Lipid Res. 2004;45:1169-1196.

10. Nonogaki K, Fuller GM, Fuentes NL,et al. Interleukin- 6 stimulates hepatic triglyceride secretion in rats. Endocrinology. 1995;136:2143-2149.

11. Ettinger WH, Varma VK, Sorci-Thomas M,et al. Cytokines decrease apolipoprotein accumulation in medium from Hep G2 cells. Arterioscler. Thromb. 1994;14:8-13.

12. Dahle DO, Mjøen G, Oqvist B, et al. Inflammation-associated graft loss in renal transplant recipients, Nephrol. Dial. Transplant. 2011;26:37563761.

13. Levey AS, Bosch JP, Lewis J, et al. A more accurate method to estimate glomerular filtration rate from serum creatinine: a new prediction equation. Modification of Diet in Renal Disease Study Group. Ann Intern Med 1999;130:461-470.

14. Levey AS, Green T, Kusek JW, Beck GJ, MDRD Study Group. A simplified equation to predict glomerular filtration rate from serum creatinine $\mathrm{J} A m$ Soc Nephrol 2000;11:155A

15. Akdis $\mathrm{M}$ et al. Interleukins, from 1 to 37 , and interferon- c: receptors, functions and roles in diseases. J Allergy Clin Immunol. 2011;127(3):701721.

16. De Serres SA, Vadivel N, Mfarrej BG, et al. Monocyte-secreted inflammatory cytokines are associated with transplant glomerulopathy in renal allograft recipients. Transplantation 2011;15;91(5):552-559.

17. Gyanendra Kumar Sonkar, Sangeeta Singh, Satyendra Kumar Sonkar, Usha Singh, Rana Gopal Singh. Evaluation of serum interleukin 6 and tumour necrosis factor alpha levels, and their association with various non-immunological parameters in renal transplant recipients. Singapore Med J 2013;54(9):511-515.

18. Gullestad L, Simonsen S, Ueland T, et al. Possible role of proinflammatory cytokines in heart allograft coronary artery disease. Am $J$ Cardiol 1999;84:999-1003. 
19. Fernández-Real JM, Broch M, Vendrell J, Rieart W. Interleukin-6 gene polymorphism and lipid abnormalities in healthy subjects. J Clin Endocrinol Metab 2000;85:1334-1339.

20. Greenberg AS, Nordan RP, Mclntosh J,et al. Interleukin-6 reduces lipoprotein lipase activity in adipose tissue of mice in vivo and in 3T3-L1 adipocytes: a possible role for interleukin-6 in cancer cachexia. Cancer
Res. 1992;52:4113-4116

21. Hingorani S, Gooley T, Pao E, Sandmaier B, McDonald G. Urinary cytokines after HCT: evidence for renal inflammation in the pathogenesis of proteinuria and kidney disease. Bone Marrow Transplant. 2014;49(3):403-409. 